\title{
ANFIS PD+I Based Hybrid Force/ Position Control of an Industrial Robot Manipulator
}

\author{
Himanshu Chaudhary, Vikas Panwar, N. Sukavanam, and Rajendra Prasad
}

\begin{abstract}
A hybrid force plus position controller based on adaptive neuro fuzzy inference system proportional derivative + integral (ANFIS-PD+I), with unspecified robot dynamics has been proposed for a robot manipulator under constrained environment. The proposed controller has been employed as a principal controller to tune up orthodox PID gains throughout the complete trajectory tracking process. The validity of the proposed controller has been studied using a 6-Degree of Freedom (DOF) PUMA robot manipulator. Simulation outcomes illustrates that the projected force / position controller adheres to the desired path closer and smoother.
\end{abstract}

Index Terms-Adaptive neuro fuzzy control, degrees of freedom, force control, position control, robot manipulator.

\section{INTRODUCTION}

An industrial robot manipulator employs desirable force normal to a given surface for following a recommended motion tangential trajectory most of the time. A Modified Hybrid Control scheme was presented in [1] which accomplished an accurate position and force control for a robot manipulator in joint space by specifying the desired compliance in Cartesian space. A hybrid neuro fuzzy (NF) position/force control with vibration suppression was presented in [2], where an adaptive neuro fuzzy inference system was applied to estimate the inverse dynamics of the space robot. The quadratic optimization and sliding-mode based hybrid position and force control approach for a robot manipulator was presented in [3] where the optimal feedback control law was derived to decide matrix differential Riccati equation and a feed forward neural network was applied to tackle the dynamic model uncertainties. A neural adaptive control scheme for hybrid force/position control of rigid robot manipulators was presented in [4]. Based on decomposed robot dynamics into force, position and redundant joint subspaces, a neural controller was proposed to tackle the parametric uncertainties, present in the dynamical model of the robot manipulator. A novel neuro-adaptive force/position tracking controller in touch with a surface influenced with non-parametric uncertainties was proposed in [5] for a robotic manipulator The configuration dependent dynamical problem

Manuscript received November 9, 2013; revised January 10, 2014.

Himanshu Chaudhary is with the Department of Electrical Engineering, IITR, India (e-mail: himan.74@ gmail.com, rpdeefee@iitr.ac.in).

Vikas Panwar and Rajendra Prasad are with the School of Vocational Studies \& Applied Sciences, GBU, India (e-mail:vikasdma@gmail.com).

N. Sukavanum is with the Department of Mathematics, IITR, India (e-mail: nskukvfma@iitr.ac.in). of the manipulator in constrained motion was dealt in [6] with the implementation of a hybrid force/velocity control for contour tracking tasks of unknown objects performed by industrial robot manipulators. An adaptive robust hybrid position/force control technique based on Lyapunov stability and bound estimation for a robot manipulator was proposed in [7]. The controller does not need the information of uncertainty bound. A position/ force controller based on the principles of invariancy for dividing of the task space into force and position subspaces was introduced in [8], to guarantee the overall stability. A hybrid impedance force / position control scheme was developed and presented in [9] for a redundant robot manipulator. The outer-loop controller improves transient performance, while the inner loop consists of a Cartesian-level potential difference controller, a redundancy resolution scheme at the acceleration level, and a joint-space inverse dynamics controller. A stable as well as generalized architecture for hybrid position/force control is presented in [10], which can influence both joint positions and torques. It was shown that kinematic instability is due to inverse of the manipulator Jacobian matrix. A robust learning control algorithm for precise path tracking of constrained robotic manipulators in the presence of state disturbances, output measurement noises and errors in initial conditions was presented in [11]. The reference [12] proposed a hybrid position, posture, force, and moment control for a six-degree-of-freedom (6-DOF) robot manipulator for the surface contact work by expansion of the conventional hybrid position and force control. The issue of hybrid adaptive network-based fuzzy inference system (ANFIS) tuning methodology based force/position control of the robot manipulators mounted on oscillatory was successfully applied in [13]. When the system is in sliding mode, force, position, and redundant joint velocity errors will approach zero irrespective of parametric uncertainties so a novel sliding-adaptive controller Based on a decomposition of the rigid robot system with motor dynamics was developed in [14], which can achieve robustness to parameter variations in both manipulator and motor. A hybrid adaptive fuzzy control approach based position/force control of robot manipulators is proposed in [15] to solve the overwhelming complexity of the deburring process and imprecise knowledge about robot manipulators. A fuzzy neural networks based Position/force controller to deal efficiently with force disturbances signals was presented in [16], to search the direction of the constraint surface for an unknown object. There are many theoretical as well as practical hurdles which are still unachievable because of unspecified robot manipulator dynamics as well as environmental complexities. Standard approaches are 
appropriate when one has the precise mathematical modeling of all the fundamental parameters vital, are available. On the other hand, most of the time one has to work in real environment where the dynamic model as well as external environmental variables are unidentified [17]. Conventional controllers like PD and PI alone do not perform well in case of removing steady state error as well as to enhance the transient response. Recently, some of the hybrid intelligent type fuzzy proportional integral derivative (FPID) controller techniques have been combined with conventional techniques and presented in [18], which illustrates the improved steady state as well as transient response. A two input fuzzy system (TIFS) based digital type proportional integral derivative (PID) controller was presented in [19]. For achieving high robustness opposed to noise, [20] accumulated twin nonlinear differentiators with a conventional fuzzy proportional and derivative (CFPD) controller to develop a different hybrid fuzzy proportional and derivative (HFPD) controller. To unravel the nonlinear control snag, [21] integrated the fuzzy gain scheduling method with a fuzzy proportional, integral and derivative (FPID) control for industrial robot manipulator To eliminates the requirement of precise dynamical models in Computed Torque Control (CTC) in the presence of structured/unstructured uncertainty, [22] combined the Fuzzy Control (FC) concept with CTC to solve the trajectory control problems of robot manipulators. The reference [23] presented a Self-Organizing Fuzzy Proportional Derivative (SOF-PD) control scheme, which exploits the simplicity and toughness of the plain PD control and enhances its gain, was offered for robot manipulators. To build and optimize the fuzzy models [24] proposed a HYFIS( Hybrid neural fuzzy Inference system), which inculcates the learning capabilities of neural networks with processing power of fuzzy logic. while considering the nonlinearities, uncertainties and external perturbations of an industrial robot manipulator, [25] proposed some new hybrid ANFIS (Adaptive neuro fuzzy inference system) control algorithms. The reference [26] applied ANFIS to address the inverse kinematic problem of determining a set of joint angles for a specific manipulator posture, while the robot's postures and trajectories were executed in Virtual Reality. An ANFIS based solution was presented in [27] to replicate the real time performance of an industrial end effector, So that the controller can facilitate dissimilar gains with disturbance signals. The reference [28] used the ANFIS for the designing of a controller based on inverse kinematic of an industrial robot manipulator.

In the present research paper, a hybrid force/position control approach is proposed for trajectory control of robot manipulator, which works well in the absence of anonymous dynamics of a robot manipulator in a constraint environment. The constraint has been taken in joint space by restricting the manipulator to move in $\mathrm{Z}$ direction. The proposed ANFIS-PD+I force/position controller follows the trajectory exceptionally smoothly with bare minimum error.

Section II establishes the actuator dynamic model for the $n$ degrees of freedom (DOF) robot manipulator. The hybrid ANFIS-PD+I force/position controller is discussed in Section III. Section IV presents simulation outcome to reveal the efficiency of the proposed controller, followed by conclusions in Section V.

\section{DYNAMICS OF ROBOT MANIPULATOR}

The traditional dynamic model of an $n$ DOF robot manipulator is presumed to be in the subsequent form[29]

$$
M(q) \ddot{q}+V_{m}(q, \dot{q})+G(q)+F(\dot{q})=\tau
$$

where $M(q) \in R^{n x n}$ represents the inertia matrix, $V_{m}(q, \dot{q}) \in \mathrm{R}^{n x n}$ represents the centripetal-coriolis matrix, $G(q) \in \mathrm{R}^{n}$ represents the gravity effects, $F(\dot{q}) \in \mathrm{R}^{n}$ denotes the friction effects, $\tau(t) \in R^{n}$ signifies the torque input control vector and $q(t), \dot{q}(t), \ddot{q}(t) \in R^{n}$ signify link position, velocity and acceleration correspondingly. If armature inductance is insignificant in the case of electric motors then the dynamics of $\mathrm{n}$ decoupled armature-controlled DC motors are

$$
J_{M} \ddot{q}_{M}+B \dot{q}_{M}+F_{M}+R \tau=K_{M} v
$$

where $v \in R^{n}$ is the control input motor voltage, $q_{M}=\operatorname{vec}\left\{q_{M i}\right\} \in R^{n}$ with $q_{M i}$ is the $\mathrm{i}^{\text {th }}$ rotor angle, motor inertia is $J_{M}$, rotor damping constant denoted by $B_{M}, F_{M}$ is the actuator friction. The actuator coefficient matrices are constants, specified as

$$
\begin{aligned}
J_{M} & =\left[\begin{array}{cc}
J_{M i} & 0 \\
0 & J_{M i}
\end{array}\right] \\
B & =\left[\begin{array}{cc}
\left(B_{M i}+K_{b i} K_{M i} / R_{a i}\right) & 0 \\
0 & \left(B_{M i}+K_{b i} K_{M i} / R_{a i}\right)
\end{array}\right] \\
R & =\left[\begin{array}{ll}
r_{i} & 0 \\
0 & r_{i}
\end{array}\right] \\
K_{M} & =\left[\begin{array}{cc}
K_{M i} / R_{a i} & 0 \\
0 & K_{M i} / R_{a i}
\end{array}\right]
\end{aligned}
$$

Here $R_{a i}$ is armature resistance, $K_{M i}$ is torque constant, $K_{b i}$ is back emf constant and $J_{M i}$ is the motor inertia of the $\mathrm{i}^{\text {th }}$ motor. $r_{i}$ is the gear ratio of the coupling

$$
\text { or } \begin{aligned}
& q_{i}=r_{i} q_{M i} \\
& q=R q_{M}
\end{aligned}
$$

where $r_{i}$ is a constant less then 1 if $q_{i}$ is revolute else units of $\mathrm{m} / \mathrm{rad}$ if $q_{i}$ is prismatic. By putting $q_{M}$ from (4) and $\tau$ from (1), the actuator dynamics for a robot manipulator may be written as

$$
\begin{aligned}
\left(J_{M}+R^{2} M\right) \ddot{q}+\left(B_{M}+R^{2} V_{m}\right) \dot{q}+ & \\
\left(R F_{M}+R^{2} F\right)+R^{2} G & =R K_{M} v
\end{aligned}
$$




$$
M^{\prime}(q) \ddot{q}+V_{m}^{\prime}(q, \dot{q})+G^{\prime}(q)+F^{\prime}(\dot{q})=K^{\prime} v
$$

where

$$
\begin{aligned}
& M^{\prime}(q)=\left(J_{M}+R^{2} M\right) ; V_{m}^{\prime}(q, \dot{q})=\left(B_{M}+R^{2} V_{m}\right) \\
& G^{\prime}(q)=R^{2} G ; F^{\prime}(\dot{q})=\left(R F_{M}+R^{2} F\right) ; K^{\prime}=R K_{M}
\end{aligned}
$$

After including nonlinear unmodelled disturbances, the (6) can be rewritten as [29]

$$
\begin{gathered}
M^{\prime}(q) \ddot{q}+V_{m}^{\prime}(q, \dot{q})+G^{\prime}(q)+F^{\prime}(\dot{q})+\tau_{d}=K^{\prime} v \\
M^{\prime}(q) \ddot{q}+N^{\prime}(q, \dot{q})+\tau_{d}=K^{\prime} v=\tau^{\prime}
\end{gathered}
$$

\section{HYBRID ANFIS-PD+I FORCE/POSITION CONTROLLER DESIGN}

A hybrid ANFIS - PD+I force/position controller applied to six DOF PUMA robot arm has been shown in Fig. 1. The ANFIS controller applied, is having two inputs and single output (TISO) structure. The inputs used are position error as well as integral error $\left(e_{m}\right)$, velocity error $\left(\dot{e}_{m}\right)$. The integral error $\left(e_{m}\right)$ has been used simply to perform orthodox integral action.

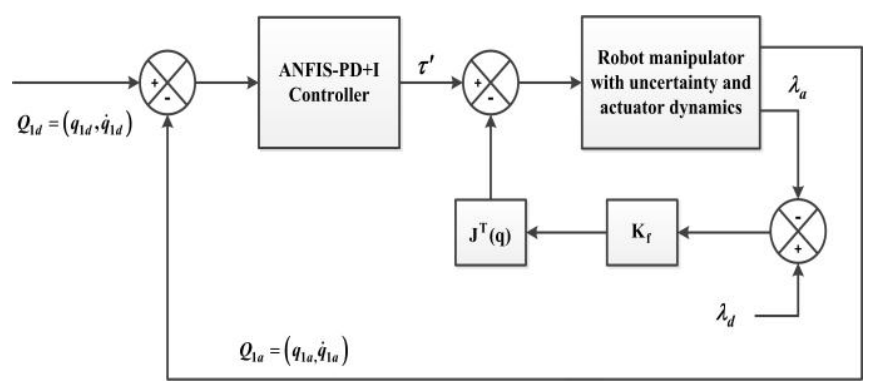

Fig. 1. ANFIS-PD+I hybrid force/position controller.

\section{A. Force/Position Controller}

The dynamic model of an $\mathrm{n}$ DOF robot manipulator with environmental contact on a prescribed surface is presumed to be in the subsequent form [29]

$$
\begin{aligned}
M^{\prime}(q) \ddot{q}+V_{m}^{\prime}(q, \dot{q})+G^{\prime}(q)+ \\
F^{\prime}(\dot{q})+\tau_{d}=\tau^{\prime}+J^{T}(q) \lambda
\end{aligned}
$$

where $q(t) \in R^{n}, J(q)$ a Jacobian matrix is related with the touching surface, and $\lambda$ is contact forces vector applied normal to the surface. The restricted surface for robot manipulator is considered in $Z$ direction in joint space. The Jacobian matrix is

$$
J(q)=\frac{\partial Z}{\partial q}
$$

The constraint equation reduces the number of degree of freedom to

$$
n_{1} \equiv n-m
$$

To find the constraint motion along the $\mathrm{Z}$ direction the reduced order dynamics for robot manipulator is

$$
\begin{aligned}
& M^{\prime}\left(q_{1}\right) L\left(q_{1}\right) \ddot{q}_{1}+V_{m}^{\prime}\left(q_{1}, \dot{q}_{1}\right) \dot{q}_{1}+ \\
& F^{\prime}\left(\dot{q}_{1}\right)+G^{\prime}\left(q_{1}\right)+\tau_{d}=\tau^{\prime}+J^{T}\left(q_{1}\right) \lambda
\end{aligned}
$$

where $q_{1}(t) \in R^{n_{1}}, L\left(q_{1}\right)$ is an extended Jacobian matrix given by

$$
L\left(q_{1}\right) \equiv\left[\begin{array}{l}
I_{n_{1}} \\
\frac{\partial Z}{\partial q_{1}}
\end{array}\right]
$$

The reduced order dynamics describing the motion in the plane of constraint surface is

$$
\bar{M} \ddot{q}_{1}+\bar{V}_{1} \dot{q}_{1}+\bar{F}+\bar{G}+\bar{\tau}_{d}=\bar{\tau}
$$

where

$$
\begin{gathered}
\bar{M}=L^{T} M^{\prime}\left(q_{1}\right) L ; \bar{V}_{1}=V_{1}\left(q_{1}, \dot{q}_{1}\right) L^{T}=L^{T}\left(V_{m}^{\prime} L+M^{\prime}\left(q_{1}\right) \dot{L}\right), \\
\bar{F}=L^{T} F^{\prime} ; \bar{G}=L^{T} G^{\prime} ; \bar{\tau}_{d}=L^{T} \tau_{d} ; \bar{\tau}=L^{T} \tau^{\prime} \\
\text { As } J\left(q_{1}\right) L\left(q_{1}\right)=0
\end{gathered}
$$

The suppositions and the necessary properties of the model given in (6) have been utilized in the proposed controller development are given as

Property 1: The inertia matrix $\bar{M}\left(q_{1}\right)$ is positive definite symmetric and bounded above and below.

Property 2: The matrix $\left(\dot{\bar{M}}\left(q_{1}\right)-2 \bar{V}_{1}\left(q_{1}, \dot{q}_{1}\right)\right)$ is skew symmetric.

Property 3: $\left\|\bar{F}\left(\dot{q}_{1}\right)\right\|=a+b\left\|\dot{q}_{1}\right\|$ for some unknown positive constants $a, b$.

Property 4: $\left\|\bar{\tau}_{d}\right\| \leq c$ for some unknown positive constants c.

\section{B. ANFIS Architecture}

It combines the learning capabilities of neural networks with processing power of fuzzy logic. [30] proposed and explained the use of ANFIS for solving a fuzzy inference system under consideration having two inputs $\boldsymbol{x}$ and $\boldsymbol{y}$ and one output $z$, which contains the rule base of two fuzzy if-then rules as

Rule 1:

$$
\text { If } x \text { is } A_{1} \text { and } y \text { is } B_{1} \text {, then } f_{1}=p_{1} x+q_{1} y+r_{1}
$$

Rule 2:

$$
\text { If } x \text { is } A_{2} \text { and } y \text { is } B_{2} \text {, then } f_{2}=p_{2} x+q_{2} y+r_{2}
$$

During the training of the network, the input is propagated layer by layer in forward direction while the error is propagated in backward direction. In layer 1, the member ship function is applied on premise parameters. To show the firing strength, layer 2 multiplies the incoming signals. Layer 3 calculates the firing strength of the signals received and 
forwards it to layer 4, which calculates an adaptive output for giving them as input to the layer 5, which computes the overall output. The procedural equations used at different layers are given in the flowchart as shown in Fig. 2.

Then the output of the hybrid ANFIS-PD+I force/position controller is, therefore

$$
u(t)=\left[L_{1}^{5}+K_{i} \int_{0}^{t} e_{m}(t) d t\right]^{*} K_{u}
$$

Here $L_{i}^{5}$ is the output ANFIS-PD controller. Thus a hybrid force position controller has the structure

$$
\tau^{\prime}=u(t)-J^{T}(q)\left[\lambda_{d}+K_{f} \tilde{\lambda}\right]
$$

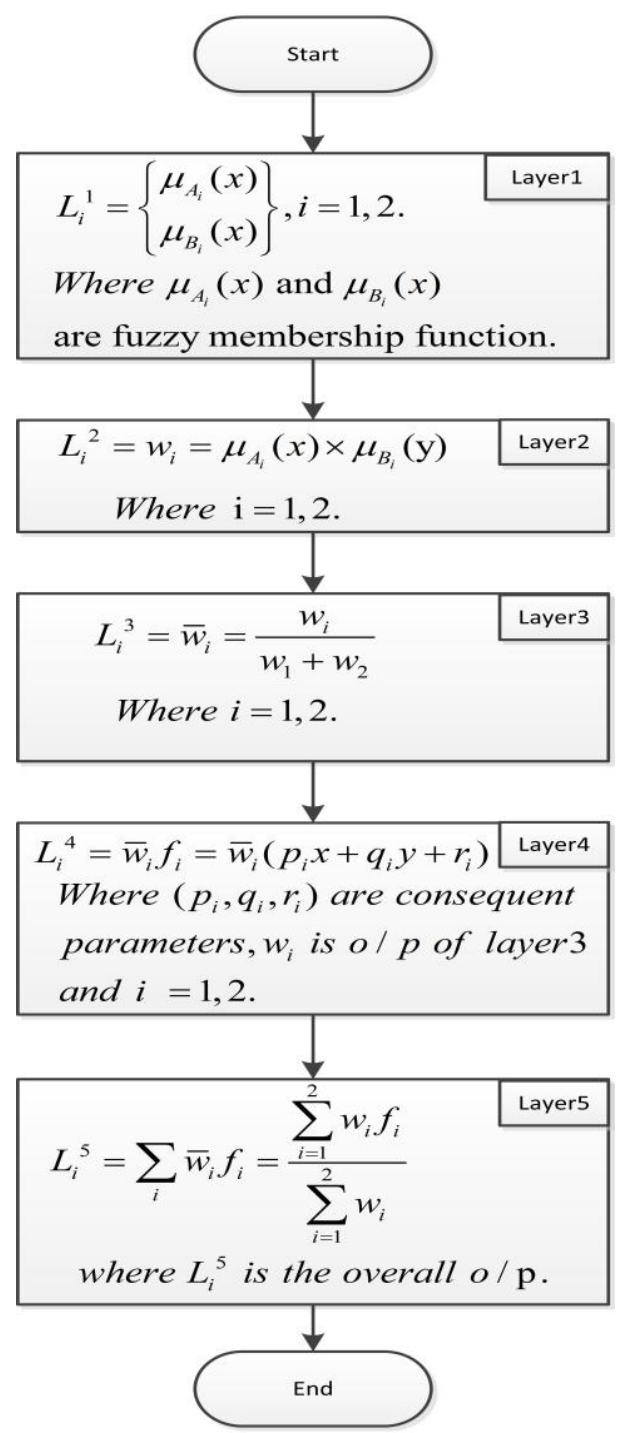

Fig. 2. Flow chart for using ANFIS procedure.

\section{Simulations AND Results}

For showing the efficiency of the suggested hybrid ANFIS-PD+I force/position controller, the simulation has been performed for a six DOF PUMA robot manipulator using MATLAB 2012b by considering the PUMA-560 robot manipulator dynamics from [31]. The following four cases have been considered to study the simulation results:

1. Complete(C): In this, we developed all the controllers based on (8), which represents the complete dynamical system including actuator dynamics.

2. Without Friction (WF): In this, we developed all the controllers based on (8), which represents the complete dynamical system including actuator dynamics. The only difference with the first case is that friction term is absent.

3. Without Gravity (WG): For this case we have designed the controllers based on a modified version of (8), which lacks of gravity term in it only.

4. Without External Disturbances (WED): controllers are developed on a modified dynamic model based on (8) for this case. The only difference from first case is the absence of external disturbances.

The working efficiency of the controller has been analyzed by the data recorded through simulation for all the cases, which is shown in the following figures. The error tracking response of hybrid ANFIS-PD + I force/position controller is shown in the Fig. 4, which clearly depicts that the proposed controller is efficient enough to track the desired trajectory with minimum steady state error.

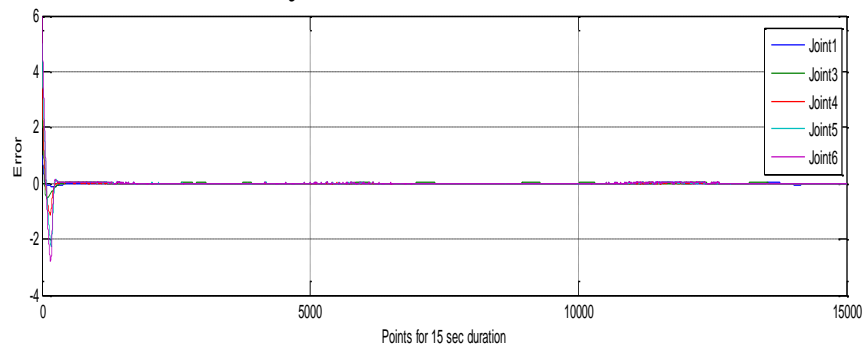

Fig. 3. Tracking error of hybrid FPD+I force/position controller.

The performance of the controller can be checked through their output response which is shown in the Fig. 5.

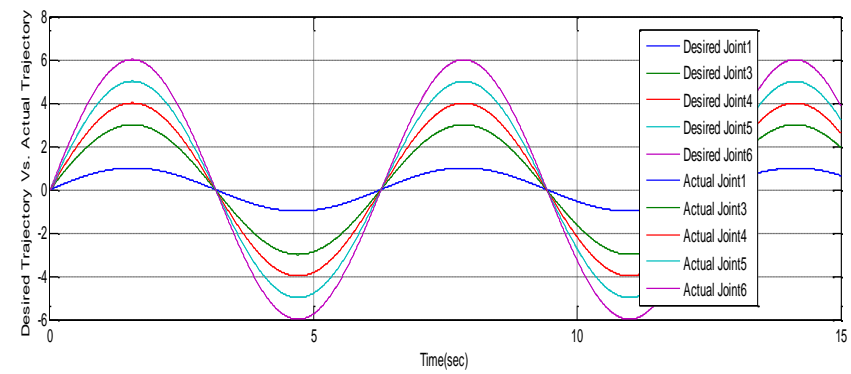

Fig. 4. Output response of hybrid ANFIS-PD+I force/position controller.

The proposed controller is able to deal with the continuous as well as discontinuous friction, gravity and other external disturbances while successfully following the desired trajectory by looking at the Fig. 5. One can see that the proposed controller is successfully following the desired trajectory with minimum errors. The simulation experiments were conducted for 24 observations with the identical gain value statistically, to study the performance of the proposed controller with other controllers. Dissimilar trajectories, dissimilar robot parameters and dissimilar simulation time $(5 \mathrm{~s}$, $10 \mathrm{~s}$, and $15 \mathrm{~s}$ ) have been employed for all 24 observations. The RMS values of path errors for joint angles $q_{1}$ to $q_{6}$ are calculated for each experiment. The RMS values average for all four cases has been calculated over 24 sets are given in Table II and Table III.

The two desired trajectories are taken for which the whole data has been recorded. The trajectories are: (I) $\sin (2 t)$ (II) $0.15 \cos \left(\left(p_{i} / 2\right) t\right)$. The friction term as well as the gravity term and external disturbances taken are as follows: 
TABLE I: RMS ERROR FOR TRAJECTORY SIN (2T)

\begin{tabular}{|c|c|c|c|c|c|c|c|c|c|c|c|c|}
\hline \multirow{2}{*}{$\begin{array}{c}\text { Joint } \\
\text { Angle }\end{array}$} & \multicolumn{4}{|c|}{5 seconds } & \multicolumn{4}{|c|}{10 seconds } & \multicolumn{4}{|c|}{15 seconds } \\
\hline & $\mathrm{C}$ & WF & WG & WED & $\mathrm{C}$ & WF & WG & WED & $\mathrm{C}$ & WF & WG & WED \\
\hline$q_{1}$ & 0.03 & 0.02 & 0.03 & 0.03 & 0.03 & 0.02 & 0.03 & 0.03 & 0.03 & 0.02 & 0.03 & 0.03 \\
\hline$q_{3}$ & 0.11 & 0.10 & 0.10 & 0.10 & 0.11 & 0.10 & 0.10 & 0.10 & 0.11 & 0.10 & 0.10 & 0.10 \\
\hline$q_{4}$ & 0.03 & 0.03 & 0.03 & 0.03 & 0.03 & 0.03 & 0.03 & 0.03 & 0.03 & 0.03 & 0.03 & 0.03 \\
\hline$q_{5}$ & 0.06 & 0.06 & 0.06 & 0.06 & 0.06 & 0.06 & 0.06 & 0.06 & 0.06 & 0.06 & 0.06 & 0.06 \\
\hline$q_{6}$ & 0.09 & 0.09 & 0.09 & 0.09 & 0.09 & 0.09 & 0.09 & 0.09 & 0.09 & 0.09 & 0.09 & 0.09 \\
\hline
\end{tabular}

TABLE II: RMS ERROR FOR TRAJECTORY $0.15 \operatorname{COS}\left(\left(P_{l} / 2\right) T\right)$

\begin{tabular}{ccccccccccccc}
\hline \hline \multirow{2}{*}{ Joint } & \multicolumn{4}{c}{5 seconds } & \multicolumn{4}{c}{ 10 seconds } & \multicolumn{4}{c}{15 seconds } \\
Angle & C & WF & WG & WED & C & WF & WG & WED & C & WF & WG & WED \\
$q_{1}$ & 0.02 & 0.02 & 0.02 & 0.02 & 0.02 & 0.02 & 0.02 & 0.02 & 0.02 & 0.02 & 0.02 & 0.02 \\
$q_{3}$ & 0.07 & 0.06 & 0.07 & 0.07 & 0.07 & 0.06 & 0.07 & 0.07 & 0.07 & 0.06 & 0.07 & 0.07 \\
$q_{4}$ & 0.06 & 0.06 & 0.06 & 0.06 & 0.06 & 0.06 & 0.06 & 0.06 & 0.06 & 0.06 & 0.06 & 0.06 \\
$q_{5}$ & 0.08 & 0.08 & 0.08 & 0.08 & 0.08 & 0.08 & 0.08 & 0.08 & 0.08 & 0.08 & 0.08 & 0.08 \\
$q_{6}$ & 0.10 & 0.10 & 0.10 & 0.10 & 0.10 & 0.10 & 0.10 & 0.10 & 0.10 & 0.10 & 0.10 & 0.10 \\
\hline \hline
\end{tabular}

$G=\left[\begin{array}{c}0 \\ -37.196 * \cos \left(t^{2}\right)-8.445 * \sin \left(t^{2}+t^{3}\right)+1.023 * \sin \left(t^{2}\right) \\ 0.248 * \cos \left(t^{2}+t^{3}\right) * \cos \left(t^{4}\right) * \sin \left(t^{5}\right)+\sin \left(t^{2}+t^{3}\right) \\ 0.028 * \sin \left(t^{2}-t^{3}\right) * \sin \left(t^{4}\right) * \sin \left(t^{5}\right) \\ 0.028 *\left[\cos \left(t^{2}-t^{3}\right) * \sin \left(t^{5}\right)\right]+\left[\sin \left(t^{2}-t^{3}\right) * \cos \left(t^{4}-t^{5}\right)\right] \\ 0\end{array}\right]$

$\tau_{d c}=\left[\begin{array}{c}q^{*} \sin 3 t+q^{\prime} \\ q^{*} 0.5 * \sin \left(2 t^{2}\right)+q^{\prime *} 1.2 * t^{2} \\ q^{*} 1.2 * \sin \left(t^{3}\right)+q^{\prime *} 0.8 * t^{3} \\ 0 \\ 0 \\ 0\end{array}\right], \tau_{d d}=\left[\begin{array}{c}0.2 * \sin t^{*} q^{\prime} \\ 0.1 * \sin \left(t^{2}\right) * q^{\prime} \\ 0.1 * \sin \left(t^{3}\right) * q^{\prime} \\ 0 \\ 0 \\ 0\end{array}\right]$

$$
F_{c}=\left[\begin{array}{c}
10 \\
5 \\
2 \\
0 \\
0 \\
0
\end{array}\right], F_{d}=\left[\begin{array}{c}
2 * \text { randomwaveform }(p i * t) \\
2 * \text { randomwaveform }(p i * t) \\
2 * \text { randomwaveform }(p i * t) \\
2 * \text { randomwaveform }(p i * t) \\
2 * \text { randomwaveform }(p i * t) \\
2 * \text { randomwaveform }(p i * t)
\end{array}\right]
$$

where $\tau_{d}=\tau_{d c}+\tau_{d d} ; F=F_{c}+F_{d}$.

The actuator parameters are:

$$
\begin{aligned}
& K_{b i}=K_{M i}=\left[\begin{array}{lllllll}
0.189 & 0.219 & 0.202 & 0.075 & 0.066 & 0.066
\end{array}\right] \\
& L_{a}^{-1}=1000 \\
& R_{a_{i}}=\left[\begin{array}{llllll}
2.1 & 2.1 & 2.1 & 6.7 & 6.7 & 6.7
\end{array}\right]
\end{aligned}
$$

Gear Ratio $=\left[\begin{array}{llllll}62.61 & 107.36 & 53.69 & 76.01 & 71.91 & 76.73\end{array}\right]$

All other parameters are taken from [31]. The PID gain parameters are taken as:

$$
\begin{aligned}
& K_{P}=\left[\begin{array}{llllll}
20 & 10 & 10 & 10 & 8 & 6
\end{array}\right] \\
& K_{D}=\left[\begin{array}{llllll}
20 & 8 & 3 & 2.0 & 2.2 & 2.0
\end{array}\right] \\
& K_{I}=\left[\begin{array}{lllllll}
0 & 2.5 & 1 & 0 & 0 & 0
\end{array}\right]
\end{aligned}
$$

The mean RMS error of the proposed ANFIS-PD+I force position controller is lower.

\section{CONCLUSION}

A hybrid ANFIS-PD+I force/position controller is suggested for the trajectory control of a robot manipulator arm with unspecified robot dynamics in real constraint environment. The system performance has been investigated extensively through simulation outcomes. The 6 DOF PUMA robot manipulator's simulation results are used to show that the proposed controller is efficient enough to handle odd situations while following the desired trajectory. The practicability of the projected controller with an appropriately defined automatic tuning of the ANFIS system using evolutionary techniques may be explored further.

\section{REFERENCES}

[1] H. Zhang and R. P. Paul, "Hybrid control of robot manipulators," in Proc. IEEE International Conference on Robotics and Automation, 1985, pp. 602-607.

[2] F. Sun, H. Zhang, and H.Wu, "Neuro-fuzzy hybrid position/force control for a space robot with flexible dual-arms," in Advances in Neural Networks - ISNN 2004, vol. 3174, F. L. Yin, J. Wang, and C. Guo, Eds., Ed. Springer Berlin Heidelberg, 2004, pp. 13-18.

[3] V. Panwar and N. Sukavanam, "Design of optimal hybrid position/force controller for a robot manipulator using neural networks," Mathematical Problems in Engineering, Hindawi Publishing Corporation, p. 23, 2007.

[4] N. Kumar, V. Panwar, N. Sukavanam, S. Sharma, and J.-H. Borm, "Neural network based hybrid force/position control for robot manipulators," International Journal of Precision Engineering and Manufacturing, vol. 12, pp. 419-426, 2011.

[5] Y. Karayiannidis, G. Rovithakis, and Z. Doulgeri, "Force/position tracking for a robotic manipulator in compliant contact with a surface using neuro-adaptive control," Automatica, vol. 43, pp. 1281-1288, 2007. 
[6] F. Jatta, G. Legnani, A. Visioli, and G. Ziliani, "On the use of velocity feedback in hybrid force/velocity control of industrial manipulators," Control Engineering Practice, vol. 14, pp. 1045-1055, 2006.

[7] I. C. Ha and M. C. Han, "Robust hybrid position/force control with adaptive scheme," JSME International Journal Series C Mechanical Systems, Machine Elements and Manufacturing, vol. 47, pp. 1161-1165, 2004.

[8] A. A. Goldenberg and P. Song, "Principles for design of position and force controllers for robot manipulators," Robotics and Autonomous Systems, vol. 21, pp. 263-277, 1997.

[9] R. V. Patel, H. A. Talebi, J. Jayender, and F. Shadpey, "A robust position and force control strategy for 7-DOF Redundant manipulators," IEEE/ASME Transactions on Mechatronics, vol. 14, pp. 575-589, 2009.

[10] W. D. Fisher and M. S. Mujtaba, "Hybrid position/force control: a correct formulation," The International Journal of Robotics Research, vol. 11, pp. 299-311, 1992

[11] D. Wang, Y. C. Soh, and C. C. Cheah, "Robust motion and force control of constrained manipulators by learning," Automatica, vol. 31, pp. 257-262, 1995.

[12] H. Qingjiu and R. Enomoto, "Hybrid position, posture, force and moment control of robot manipulators," in Proc. IEEE International Conference on Robotics and Biomimetics, 2008, 2009, pp. 1444-1450.

[13] J. Lin, C. C. Lin, and H. Lo, "Hybrid position/force control of robot manipulators mounted on oscillatory bases using adaptive fuzzy control," in Proc. IEEE International Symposium on Intelligen Control, 2010, pp. 487-492.

[14] C. M. Kwan, "Hybrid force/position control for manipulators with motor dynamics using a sliding-adaptive approach," IEEE Transactions on Automatic Control, vol. 40, pp. 963-968, 1995.

[15] H. Feng-Yi and F. Li-Chen, "Intelligent robot deburring using adaptive fuzzy hybrid position/force control," IEEE Transactions on Robotics and Automation, vol. 16, pp. 325-335, 2000.

[16] K. Kiguchi and T. Fukuda, "Position/force control of robot manipulators for geometrically unknown objects using fuzzy neural networks," IEEE Transactions on Industrial Electronics, vol. 47, pp. 641-649, 2000

[17] O. Castillo and P. Melin, "Intelligent adaptive model-based control of robotic dynamic systems with a hybrid fuzzy-neural approach," Applied Soft Computing, Elsevier, vol. 3, pp. 363-378, 2003.

[18] G. M. Khoury, M. Saad, H. Y. Kanaan, and C. Asmar, "Fuzzy PID Control of a Five DOF Robot Arm," Journal of Intelligent and Robotic Systems, Springer, vol. 40, pp. 299-320, 2004.

[19] W. Li, X. G. Chang, J. Farrell, and F. M. Wahl, "Design of an enhanced hybrid fuzzy P+ID controller for a mechanical manipulator," IEEE Transactions on Systems, Man, and Cybernetics, vol. 31, pp. 938-945, 2001.

[20] Y. X. Su, S. X. Yang, D. Sun, and B. Y. Duan, "A simple hybrid fuzzy PD controller," Mechatronics, vol. 14, pp. 877-890, 2004.

[21] S. Ya Lei and E. Meng Joo, "Hybrid fuzzy control of robotics systems," IEEE Transactions on Fuzzy Systems, vol. 12, pp. 755-765, 2004.

[22] Z. Song, J. Yi, D. Zhao, and X. Li, "A computed torque controller for uncertain robotic manipulator systems: Fuzzy approach," Fuzzy Sets Systems, Elsevier, vol. 154, pp. 208-226, 2005.

[23] F. Salas, M. Llama, and V. Santibanez, "A stable self-organizing Fuzzy PD control for robot manipulators," International Journal of Innovative Computing, Information and Control, vol. 9, 2013.

[24] J. Kim and N. Kasabov, "HyFIS: adaptive neuro-fuzzy inference systems and their application to nonlinear dynamical systems," Neural Networks, vol. 12, pp. 1301-1319, 1999.

[25] S. Alavandar and M. J. Nigam, "New hybrid adaptive neuro-fuzzy algorithms for manipulator control with uncertainties-Comparative study," ISA Transactions, vol. 48, pp. 497-502, 2009.

[26] Y. I. A. Mashhadany, "ANFIS-Inverse-Controlled PUMA 560 Workspace Robot with Spherical Wrist," Procedia Engineering, vol. 41, pp. 700-709, 2012.

[27] M. P. F. Queen, M. S. Kumar, and P. B. Aurtherson, "ANFIS technique applied to the control of a robot manipulator with disturbances," Procedia Engineering, vol. 38, pp. 1984-1993, 2012.

[28] C. Himanshu and P. Rajendra, "Intelligent inverse kinematic control of SCORBOT-ER VPlus robot manipulator," International Journal of Advances in Engineering \& Technology, vol. 1, pp. 158-169, 2011.
[29] F. L. Lewis, S. Jagannathan, and A. Yesildirek, Neural Network Control of Robot Manipulators and Non-Linear Systems, 1st ed. UK: Taylor \& Francis, 1999.

[30] J. S. R. Jang, "ANFIS: adaptive-network-based fuzzy inference system," IEEE Transactions on Systems, Man and Cybernetics, vol. 23, pp. 665-685, 1993.

[31] B. Armstrong, O. Khatib, and J. Burdick, "The explicit dynamic model and inertial parameters of the PUMA 560 arm," in Proc. IEEE Int Conf. on Robotics and Automation, 1986, pp. 510-518.

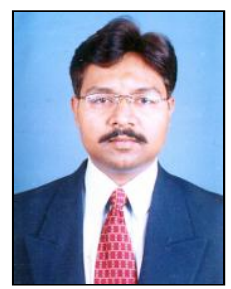

Himanshu Chaudhary received his B.E. in electronics and telecommunication from Amravati University, Amravati, India in 1996, M.E. in automatic controls and robotics from M.S. University, Baroda, Gujarat, India in 2000. Presently he is a research scholar in Electrical Engineering Department, IIT Roorkee, India. His area of interest includes industrial robotics, computer networks and embedded systems.

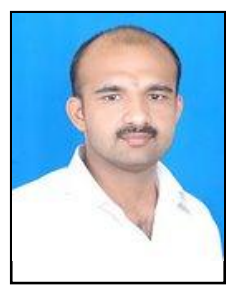

Viaks Panwar received B.Sc. (mathematics) degree from Meerut University, India in 1998. He received M.Sc. and Ph.D. degree in mathematics from the IIT Roorkee, India in 2000 and 2008 respectively. He also served as an assistant professor (July $312004-9$ March 2010) at Chaudhary Devi Lal University Sirsa, Haryana. He also worked as an assistant professor (March 102010 - 16 August 2010) at Defence Institute of Advanced Technology, (Deemed University) Pune, Maharashtra. Presently he is working as an assistant professor from 18 August 2010 at Gautam Buddha University, Greater Noida, Uttar Pradesh.

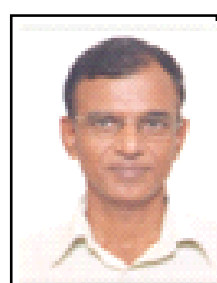

N. Sukavanum was born in India in 1957. He received B.Sc. (Maths) degree from University of Madras, India in 1977 and M.Sc. (Maths) from the same university in 1979 and Ph.D. (Maths) from IISC Banglore, India in 1985 respectively. He has served as a scientist-B in Naval Science and Technological Laboratory, DRDO, Vizag, and as a research scientist in I I T, Bombay. He also worked as a lecturer at Birla Institute of Technology and Science, Pilani, Rajasthan from 1990 to 1996 . He worked as an assistant professor in Mathematics Department at Indian Institute of Technology, Roorkee during May 1996-April 2004 Presently, he is a professor in the Department of Mathematics, Indian Institute of Technology Roorkee, Roorkee (India). He has published 60 papers in various journals/conferences. He has guided nine Ph.D's, and presently six $\mathrm{Ph}$. D's are under progress. His research interests include nonlinear analysis, control theory and robotics and control.

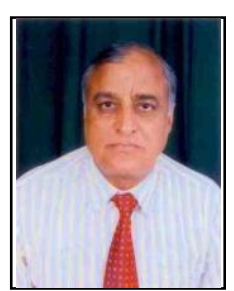

Rajendra Prasad received B.Sc. (Hons.) degree from Meerut University, India in 1973. He received B.E. M.E. and Ph.D. degree in electrical engineering from the University of Roorkee, India in 1977, 1979 and 1990 respectively.. He also served as an assistant engineer in Madhya Pradesh Electricity Board (MPEB) from 1979- 1983. Currently, he is a professor in the Department of Electrical Engineering, Indian Institute of Technology Roorkee, Roorkee (India). He has more than 32 years of experience of teaching as well as industry. He has published 176 papers in various journals/conferences and received eight awards on his publications in various national/international journals/conferences proceeding papers. He has guided Seven PhD's, and presently six PhD's are under progress. His research interests include control, optimization, system engineering and model order reduction of large scale systems and industrial robotics. 